\title{
Marcadores da autopercepção positiva de saúde de pessoas idosas no Brasil
}

Positive self-perceived health markers in the older adult population in Brazil

Marcadores de la autopercepción positiva de la salud de personas mayores en Brasil

\author{
Marília Miranda Forte Gomes ${ }^{1}$ ib https://orcid.org/0000-0001-8584-9676 \\ Luiz Alexandre Rodrigues da Paixão ${ }^{1}$ in https://orcid.org/0000-0003-0775-5935 \\ Andrea Mathes Faustino ${ }^{1}$ io https: /orcid.org/0000-0002-5474-7252 $^{2}$ \\ Rebeca Carmo de Souza Cruz ${ }^{1}$ io https://orcid.org/0000-0002-2030-3434 \\ Leides Barroso Azevedo Moura' id https://orcid.org/0000-0002-1208-4569
}

Como citar:

Gomes MM, Paixão LA, Faustino AM, Cruz RC, Moura LB. Marcadores da autopercepção positiva de saúde de pessoas idosas no Brasil. Acta Paul Enferm. 2021;34:APE02851.

DOI

http://dx.doi.org/10.37689/actaape/2021A002851

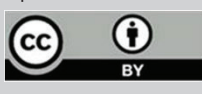

Envelhecimento; Autoimagem; Idoso; Determinantes sociais da

saúde; Brasil

Keywords

Aging; Population health; Self concept; Aged Social determinants of health; Brazi

Descriptores

Envejecimiento; Salud poblacional; Autoimagen; Anciano; Determinantes sociales de la salud; Brasil

Submetido 15 de Outubro de 2019

Aceito

1 de Junho de 2020

Autor correspondente Marilia Miranda Forte Gomes E-mail: mariliamfg@gmail.com

\section{Resumo}

Objetivo: Analisar os determinantes demográficos e socioeconômicos que podem influenciar a autopercepção positiva de saúde de pessoas idosas no Brasil.

Métodos: Estudo quantitativo de natureza descritiva que utiliza dados da Pesquisa Nacional de Saúde realizada em 2013 que constituí uma amostra ponderada de 11,8 milhões de idosos residentes no Brasil. A variável desfecho analisada é a autopercepção de saúde e foi categorizada em positiva e negativa. As variáveis independentes contemplam três dimensões: sociodemográficas, estilo de vida e aspectos de saúde. As análises foram apresentadas em forma de razão de chances obtidas através da aplicação do modelo de regressão logístico binário.

Resultado: Os resultados evidenciam, tanto para homens quanto para mulheres, que ter se autodeclarado branco, não apresentar doenças crônicas ou incapacidades funcionais, ter um estilo de vida mais saudável (nunca ter fumado e ter participado de atividades de interação social religiosa com maior frequência), e níveis de escolaridade mais elevados contribuem para que as chances sejam maiores de uma percepção positiva da saúde. Pessoas idosas com ensino médio completo e superior incompleto apresentaram três vezes maiores chances de percepção positiva da saúde em relação aos idosos sem o nível fundamental completo.

Conclusão: 0 estudo identificou os determinantes sociais de saúde dos idosos e a relação com uma percepção positiva da saúde. Identificar e analisar estas associações são pontos importantes para a elaboração de políticas públicas específicas, visando a equidade e a promoção da saúde.

\section{Abstract}

Objective: To analyze the demographic and socioeconomic determinants that can influence the positive selfperceived health of older adult people in Brazil.

Methods: A quantitative and descriptive study using data from the Brazilian National Health Survey conducted in 2013. This survey constituted a weighted sample of 11.8 million older people living in Brazil. The outcome variable analyzed is self-perceived health, and was categorized as positive and negative. The independent variables contemplate three dimensions: sociodemographic, lifestyle and health aspects. The analyzes were presented as Odds Ratio obtained by applying the binary logistic regression model.

Results: The results show, for both men and women, that having declared themselves white, not having chronic illnesses or functional disabilities, having a healthier lifestyle (never having smoked and having participated in religious social interaction activities more frequently) and higher levels of education contribute to the chances of a better perception of health being greater. Older people with complete high school and incomplete higher education were three times more likely to have a positive perception of health than those without complete elementary school. 
Conclusion: This study identified the social determinants of health of older persons and the relationship with a positive perception of health. Identifying and analyzing these associations are important points for the elaboration of specific public policies, aiming at equity and health promotion.

\section{Resumen}

Objetivo: Analizar los determinantes demográficos y socioeconómicos que pueden influir en la autopercepción positiva de la salud de personas mayores en Brasil. Métodos: Estudio cuantitativo de naturaleza descriptiva que utiliza datos de la Encuesta Nacional de Salud realizada en 2013, que constituye una muestra ponderada de 11,8 millones de adultos mayores residentes en Brasil. La variable de resultado analizada es la autopercepción de la salud y fue categorizada en positiva y negativa. Las variables independientes contemplan tres dimensiones: sociodemográficas, estilo de vida y aspectos de la salud. Los análisis se presentaron en forma de razón de momios obtenidos mediante la aplicación del modelo de regresión logística binario.

Resultado: Los resultados evidencian que, tanto en hombres como en mujeres, haberse autodeclarado blanco, no presentar enfermedades crónicas o incapacidades funcionales, tener un estilo de vida más saludable (nunca haber fumado y haber participado en actividades de interacción social religiosa con mayor frecuencia) y tener un nivel de escolaridad más elevado contribuyen para que las probabilidades de una percepción positiva de la salud sean mayores. Personas mayores con educación secundaria completa y educación superior incompleta presentaron probabilidades tres veces mayores de una percepción positiva de la salud con relación a los adultos mayores sin el nivel primario completo.

Conclusión: El estudio identificó los determinantes sociales de salud de los adultos mayores y la relación con una percepción positiva de la salud. Identificar y analizar estas relaciones son puntos importantes para la elaboración de políticas públicas específicas, que busquen equidad y promoción de la salud.

\section{Introdução}

O envelhecimento da população caracteriza o aumento da longevidade e é considerado pela Organização Mundial de Saúde como uma história de sucesso das políticas de saúde e de desenvolvimento socioeconômico. ${ }^{(1)}$ A recente história social da longevidade é marcada pela heterogeneidade das condiçôes sociais de produção da saúde, que envolve trajetórias desiguais entre as pessoas e seus processos de envelhecimento ao longo do curso da vida. (2) A ampliação da longevidade apresenta oportunidades e desafios, incluindo a necessidade de captar o estado de saúde da população. ${ }^{(2,3)}$

Uma das formas de delinear o quadro geral de saúde da populaçáo idosa constitui-se pelo uso da autopercepção de saúde como um indicador que revela o estado dos indivíduos, considerando-os integralmente seus aspectos físicos, comportamentais e emocionais, bem como a percepção de satisfação com suas próprias vidas. ${ }^{(4-6)}$

A autopercepçáo da saúde tem sido usada como indicador válido acerca do estado de saúde em pesquisas envolvendo a população idosa. ${ }^{(7,8)}$ Mesmo dotada de subjetividades, tal indicador para a pessoa idosa está relacionada com medidas objetivas e tem sido utilizado como preditor da mortalidade e do declínio funcional..$^{(9,10)}$

A Pesquisa Nacional de Saúde (PNS) capta de maneira inédita alguns aspectos relacionados às condiçôes da população brasileira, abordando, entre outras questôes, a percepção individual da saúde em múlti- plas dimensões, que é foco deste trabalho. ${ }^{(11)}$ Estudos que abordam a autopercepçáo da saúde e qualidade de vida revelam que as condições socioeconômicas e os laços sociais influenciam na percepção negativa da saúde, ${ }^{(12,13)}$ entretanto a PNS também oferece dados nacionais que permitem a elaboração de novas análises dessas circunstâncias socioeconômicas, psicossociais e comportamentais de pessoas idosas e a relação com a avaliaçáo positiva da saúde. A caracterização nacional dos determinantes da percepção positiva da saúde por parte dos idosos brasileiros representa uma lacuna na literatura brasileira.

Para a promoçấo de um envelhecimento ativo é necessário considerar os fatores que agem concomitantemente e que podem estar associados à percepção positiva da saúde. $O$ presente trabalho assume que as desigualdades sociais no Brasil e no mundo geram determinantes sociais das desigualdades em saúde da população. ${ }^{(2-4)}$ Neste sentido, o modo como as pessoas idosas avaliam a saúde carece de ser decifrado a partir de uma perspectiva teórica de gradiente social, onde se assume que posiçôes sociais distintas produzem diferenciais de saúde e revelam a "causa das causas" das iniquidades em saúde. ${ }^{(14)}$ Pesquisas com esse tipo de abordagem apoiam a elaboraçáo de indicadores para monitoramento de políticas públicas e ação pública voltadas para $\mathrm{o}$ envelhecimento ao longo da vida e aprofundam a compreensão dos profissionais da saúde acerca dos determinantes da longevidade.

Neste sentido, o estudo objetiva analisar os determinantes demográficos e socioeconômicos que 
podem influenciar a autopercepção positiva de saúde de pessoas idosas no Brasil.

\section{Métodos}

Trata-se de uma pesquisa quantitativa, de natureza descritiva, que utilizou dados secundários da Pesquisa Nacional de Saúde (PNS). A PNS é uma pesquisa de inquérito domiciliar de base populacional com abrangência nacional, realizada em 2013, pelo Instituto Brasileiro de Geografia e Estatística (IBGE) em parceria com a Secretaria de Vigilância Sanitária do Ministério da Saúde e a Fundação Oswaldo Cruz que teve como foco abordar as doenças crônicas, estilos de vida e acesso a serviços de saúde. ${ }^{(11)}$

Dada a riqueza de informaçóes dessa base de dados, o referencial teórico e o objetivo proposto neste estudo, selecionou-se as seguintes questóes: autopercepção da saúde da pessoa idosa, variáveis sociodemográficas, de estilo de vida e de aspectos da saúde, conforme itens do questionário individual respondido pela população com 60 anos ou mais.

As características sociodemográficas selecionadas foram: idade; cor/etnia autodeclarada, sendo categorizada em brancos, negros (pretos e pardos) e outros (amarelos e indígenas); região geográfica de residência; e nível de escolarização.

As variáveis acerca do estilo de vida selecionadas para o estudo foram: consumo de bebidas alcoólicas; tabagismo (uso de charutos, cigarrilha, cachimbo, narguilé); e, participação, no último ano, em atividades religiosas.

Outra variável investigada se refere à presença de Doenças Crônicas Não Transmissíveis (DCNT) diagnosticadas: hipertensão, diabetes, colesterol alto, doenças do coraçáo, derrame ou acidente vascular cerebral, asma ou bronquite asmática, artrite ou reumatismo, problemas de coluna, doença do pulmão, câncer e insuficiência renal crônica. As categorias utilizadas foram: não possui doença alguma; possui uma doença dentre as consideradas; possui de duas a três; e possui mais de três.

A capacidade funcional dos idosos foi caracterizada pela identificação de dificuldades em execu- tar tarefas classificadas como Atividades Básicas da Vida Diária (AVD) e Atividades Instrumentais da Vida Diária (AIVD). As AVD consistem em: comer e beber com um prato colocado à sua frente, incluindo segurar um garfo, cortar alimentos e beber em um copo; tomar banho, incluindo entrar e sair do chuveiro ou banheira; ir ao banheiro sozinho, incluindo sentar-se e levantar-se do vaso sanitário; vestir-se, incluindo calçar meias e sapatos, abrir e fechar o zíper e botóes; andar em casa sozinho em um mesmo andar; deitar ou levantar-se da cama; sentar ou levantar-se da cadeira.

As AIVD indicadas foram: fazer compras de alimentos, roupas ou medicamentos; cuidar das próprias finanças; tomar remédios; ir ao médico; utilizar transportes como ônibus, metrô, táxi ou carro. Idosos que não conseguiam executar pelo menos uma das atividades de forma autônoma ou que tinham pequena ou muita dificuldade em qualquer uma delas foram considerados como dependentes; aqueles que não tinham dificuldade, como independentes.

A variável dependente foi a autoavaliação de saúde, contemplada pela questão: "Em geral como o(a) sr(a). avalia a sua saúde?”. Para o presente estudo, as alternativas de respostas do questionário da PNS foram agregadas em duas categorias: (1) positiva - muito boa, boa - e (0) negativa - ruim e muito ruim. Optou-se por náo incluir na amostra as pessoas idosas que responderam "regular", uma vez que essa resposta não permitiria escolher adequadamente um parâmetro de discriminação que diferenciaria a percepção dos respondentes na direção do polo positivo ou do negativo e considerada como ponto neutro para fins do presente estudo. Os entrevistados de escolaridade mais baixa, especialmente em países marcados pela intensa desigualdade social, podem escolher ponto neutro para suas respostas em detrimento da dificuldade de compreensão das questóes e não necessariamente de uma posição acerca da questão perguntada. ${ }^{(15)}$

Neste trabalho, adotou-se o processo stepwise de inclusão das variáveis e definiram-se dois modelos um para cada sexo - com as variáveis que tiveram significância para a percepção positiva do estado de saúde do idoso. 
Para a análise da associação entre a autopercepção de saúde e os determinantes sociais - variáveis independentes -, foi utilizada a regressão logística binária, buscando identificar, em termos de razão de chances o grau de associação existente entre as variáveis dependente e independentes, o que aponta se o determinante funciona como possível fator de risco ou de proteção para a autopercepção positiva da saúde do idoso. Para a obtenção dos modelos propostos e análise descritiva dos dados, utilizaram-se rotinas específicas para o tratamento de dados provenientes de amostras complexas disponíveis no programa SPSS, resultando assim em uma amostra ponderada de 26,4 milhóes de idosos.

O projeto da PNS foi aprovado na Comissão Nacional de Ética em Pesquisa, conforme o Processo $\mathrm{n}^{\circ} 328.159$, de 26 de junho de 2013, e os dados encontram-se públicos e publicizados no site do IBGE (www.ibge.gov.br). Dessa maneira, conforme Resolução 466/2012 e 510/2016 do Conselho Nacional de Saúde, por se tratar de um estudo que utiliza dados secundários que não contém identificação de seus participantes não se fez necessário submeter novamente a presente pesquisa para análise em comitê de ética.

\section{Resultados}

O segmento composto por pessoas de 60 anos ou mais, que perceberam a sua saúde como positiva (muito boa ou boa) ou negativa (ruim ou muito ruim) compreendeu 11,8 milhóes de indivíduos, em 2013, pela PNS, correspondendo a 6\% dos respondentes totais da PNS. Destes, a sua maioria era composta por $56 \%$ de mulheres, residiam nas regióes Centro-Oeste (50\%) e Nordeste (23\%), tinham idade até 69 anos (54\%), autodeclararam-se brancos (54\%) e possuíam nível de escolaridade não superior ao fundamental incompleto (64\%). Entre aqueles do sexo masculino que perceberam sua saúde como positiva, observou-se, conforme mostra a tabela 1 , que são idosos jovens (32\%), de cor/etnia branca $(58 \%)$ e que residem principalmente na Região Centro-Oeste (53\%). Destaca-se o percentual daqueles que autodeclararam ter nível superior completo (18\%). No que diz respeito às variáveis de estilo de vida (Tabela 2), a maioria declarou ser ex fumante $(44 \%)$ e frequentar atividades religiosas (42\%). O percentual de idosos que fazem uso de bebidas alcoólicas é maior nesse grupo que avaliou como muito boa ou boa a sua saúde do que entre aqueles que avaliaram de maneira negativa ( $47 \%$ e $22 \%$, respectivamente). Conforme esperado (Tabela 3), a população masculina com 60 anos ou mais que não apresenta nenhuma incapacidade e com nenhuma ou apenas uma DCNT apresentou maior percentual de saúde percebida como positiva (99\% e $72 \%$, respectivamente). Valores semelhantes foram vislumbrados entre as mulheres idosas que avaliariam positivamente a sua saúde. No geral (Tabela 1 ), a maioria residia no Centro-Oeste $(55 \%)$, possuía idade até 64 anos (37\%), era branca $(59 \%)$ e tinha nível superior completo $(16 \%)$. Observou-se também que o referido grupo foi constituído por

Tabela 1. Percentual de idosos, de acordo com 0 sexo, segundo a autopercepção da saúde e variáveis sociodemográficas $(n=11871947)$

\begin{tabular}{|c|c|c|c|c|c|c|}
\hline \multirow{3}{*}{$\begin{array}{l}\text { Variáveis } \\
\text { Sóciodemográficas }\end{array}$} & \multicolumn{6}{|c|}{ Sexo/Autopercepção da saúde } \\
\hline & \multicolumn{3}{|c|}{ Masculino } & \multicolumn{3}{|c|}{ Feminino } \\
\hline & $\begin{array}{c}\text { Positiva } \\
\%\end{array}$ & $\begin{array}{c}\text { Negativa } \\
\%\end{array}$ & $\begin{array}{c}\text { Total } \\
\%\end{array}$ & $\begin{array}{c}\text { Positiva } \\
\%\end{array}$ & $\begin{array}{c}\text { Negativa } \\
\%\end{array}$ & $\begin{array}{c}\text { Total } \\
\%\end{array}$ \\
\hline \multicolumn{7}{|l|}{ Região } \\
\hline Norte & 5 & 10 & 6 & 4 & 6 & 5 \\
\hline Nordeste & 20 & 33 & 23 & 19 & 38 & 23 \\
\hline Sudeste & 15 & 14 & 15 & 16 & 15 & 16 \\
\hline Sul & 7 & 7 & 7 & 6 & 7 & 6 \\
\hline Centro-Oeste & 53 & 35 & 50 & 55 & 34 & 50 \\
\hline \multicolumn{7}{|l|}{ Faixa etária } \\
\hline 60 a 64 anos & 32 & 27 & 31 & 37 & 30 & 35 \\
\hline 65 a 69 anos & 31 & 32 & 31 & 29 & 30 & 29 \\
\hline 70 a 74 anos & 17 & 11 & 16 & 13 & 15 & 13 \\
\hline 75 a 79 anos & 11 & 18 & 12 & 13 & 13 & 13 \\
\hline Mais de 80 anos & 9 & 13 & 10 & 8 & 11 & 9 \\
\hline \multicolumn{7}{|l|}{ Raça/cor } \\
\hline Brancos & 58 & 30 & 53 & 59 & 39 & 55 \\
\hline Negros & 40 & 70 & 45 & 39 & 59 & 44 \\
\hline Amarelos/Indígenas & 2 & 0 & 2 & 1 & 2 & 2 \\
\hline \multicolumn{7}{|l|}{ Nível de instrução } \\
\hline $\begin{array}{l}\text { Até fundamental } \\
\text { Incompleto }\end{array}$ & 60 & 88 & 65 & 58 & 86 & 64 \\
\hline $\begin{array}{l}\text { Fundamental } \\
\text { completo e Médio } \\
\text { Incompleto }\end{array}$ & 9 & 6 & 8 & 8 & 4 & 7 \\
\hline $\begin{array}{l}\text { Médio completo e } \\
\text { Superior Incompleto }\end{array}$ & 13 & 5 & 12 & 18 & 7 & 15 \\
\hline Superior completo & 18 & 1 & 15 & 16 & 3 & 13 \\
\hline Total & 100 & 100 & 100 & 100 & 100 & 100 \\
\hline
\end{tabular}

Notas: Considerando apenas os casos em que o próprio idoso era o informante.

Percepção positiva compreende as categorias Muito boa e Boa; Percepção negativa compreende as categorias Ruim e Muito ruim. 
Tabela 2. Percentual de idosos, de acordo com o sexo, segundo autopercepção da saúde e variáveis de estilo de vida $(\mathrm{n}=11.871 .947)$

\begin{tabular}{|c|c|c|c|c|c|c|}
\hline \multirow{3}{*}{$\begin{array}{l}\text { Variáveis de estilo } \\
\text { de vida }\end{array}$} & \multicolumn{6}{|c|}{ Sexo/Autopercepção da saúde } \\
\hline & \multicolumn{3}{|c|}{ Masculino } & \multicolumn{3}{|c|}{ Feminino } \\
\hline & $\begin{array}{c}\text { Positiva } \\
\%\end{array}$ & $\begin{array}{c}\text { Negativa } \\
\%\end{array}$ & $\begin{array}{c}\text { Total } \\
\%\end{array}$ & $\begin{array}{c}\text { Positiva } \\
\%\end{array}$ & $\begin{array}{c}\text { Negativa } \\
\% \\
\end{array}$ & $\begin{array}{c}\text { Total } \\
\% \\
\end{array}$ \\
\hline \multicolumn{7}{|l|}{ Bebidas alcóolicas } \\
\hline Não consome & 53 & 78 & 58 & 81 & 94 & 84 \\
\hline $\begin{array}{l}\text { Menos de } 1 \text { vez } \\
\text { por mês }\end{array}$ & 12 & 10 & 12 & 9 & 2 & 7 \\
\hline $\begin{array}{l}\text { Uma ou mais vezes } \\
\text { por mês }\end{array}$ & 35 & 12 & 31 & 10 & 4 & 9 \\
\hline \multicolumn{7}{|l|}{ Tabagismo } \\
\hline Nunca fumou & 41 & 32 & 40 & 70 & 64 & 68 \\
\hline Fumante & 15 & 23 & 17 & 11 & 12 & 11 \\
\hline Ex-fumante & 44 & 44 & 44 & 19 & 24 & 21 \\
\hline \multicolumn{7}{|l|}{ Atividades religiosas } \\
\hline Não frequenta & 37 & 38 & 37 & 20 & 30 & 22 \\
\hline Raramente frequenta & 22 & 24 & 22 & 16 & 17 & 16 \\
\hline Frequentemente & 42 & 38 & 41 & 65 & 52 & 62 \\
\hline Total & 100 & 100 & 100 & 100 & 100 & 100 \\
\hline
\end{tabular}

Fonte: Microdados da PNS 2013. Elaboração própria.

Notas: Considerando apenas os casos em que o próprio idoso era o informante.

Percepção positiva compreende as categorias Muito boa e Boa; Percepção negativa compreende as categorias Ruim e Muito ruim.

Tabela 3. Percentual de idosos, de acordo com o sexo e autopercepção da saúde, segundo aspectos de saúde $(\mathrm{n}=11.871 .947)$

\begin{tabular}{lccccccc}
\hline Quantidade de & \multicolumn{5}{c}{ Sexo/Autopercepção da saúde } \\
\cline { 2 - 4 } $\begin{array}{l}\text { doenças crônicas } \\
\text { não transmissíveis e } \\
\text { Capacidade Funcional }\end{array}$ & $\begin{array}{c}\text { Masculino } \\
\text { Positiva } \\
\text { \% }\end{array}$ & $\begin{array}{c}\text { Negativa } \\
\%\end{array}$ & $\begin{array}{c}\text { Total } \\
\%\end{array}$ & $\begin{array}{c}\text { Positiva } \\
\%\end{array}$ & $\begin{array}{c}\text { Negativa } \\
\%\end{array}$ & $\begin{array}{c}\text { Total } \\
\%\end{array}$ \\
\hline $\begin{array}{l}\text { Doenças crônicas não } \\
\text { transmissíveis }\end{array}$ & & & & & & & \\
$\quad$ Nenhuma & 39 & 12 & 34 & 32 & 4 & 26 \\
$\quad$ Uma & 32 & 32 & 32 & 30 & 14 & 27 \\
$\quad$ Duas a três & 25 & 37 & 27 & 31 & 46 & 35 \\
$\quad$ Quatro ou mais & 4 & 18 & 7 & 6 & 36 & 13 \\
Capacidade funcional & & & & & & \\
$\quad$ Dependente AIVD & 1 & 13 & 3 & 4 & 15 & 7 \\
$\quad$ Dependente AVD e AIVD & 0 & 1 & 0 & 0 & 3 & 1 \\
$\quad$ Independente & 99 & 86 & 97 & 96 & 81 & 93 \\
Total & 100 & 100 & 100 & 100 & 100 & 92,5 \\
\hline
\end{tabular}

Fonte: Microdados da PNS 2013. Elaboração própria.

Notas: Considerando apenas os casos em que o próprio idoso era o informante.

Percepção positiva compreende as categorias Muito boa e Boa; Percepção negativa compreende as categorias Ruim e Muito ruim.

mulheres com estilo de vida mais saudável - (Tabela 2) $(70 \%$ nunca fumou e $65 \%$ frequentava atividades religiosas). Fazer uso de bebida alcoólica, assim como entre os homens, é maior entre àquelas com saúde declarada como positiva (19\%) do que entre àquelas que declararam como negativa $(6 \%)$. O percentual de mulheres independentes (96\%), como nenhuma ou uma doença crônica não transmissível (62\%) finalizam as características investigadas para esse grupo com autopercepção da saúde positiva
(Tabela 3). A tabela 4 apresenta as razóes de chances para a autopercepção de saúde, considerando os determinantes sociais que permaneceram para os modelos finais, após o processo stepwise. Observou-se que a variável idade não foi significativa, independentemente do sexo. No geral, tanto para homens quanto para mulheres, ter se autodeclarado branco, não apresentar DCNT ou incapacidades funcionais, e ter um estilo de vida mais saudável, contribuiu para que as chances sejam maiores de uma autopercepçáo da saúde positiva (conforme RC menores que um e que são apresentadas na tabela 4). Entre os homens residentes nas regióes Norte

Tabela 4. Razões de Chances (RC) do modelo de regressão logística binário final para autopercepção de saúde e determinantes sociais, segundo o sexo

\begin{tabular}{|c|c|c|c|c|}
\hline \multirow{3}{*}{ Variáveis } & \multicolumn{4}{|c|}{$\begin{array}{l}\text { Modelo final - idosos do sexo masculino e } \\
\text { feminino }\end{array}$} \\
\hline & \multicolumn{2}{|c|}{$\begin{array}{l}\text { Modelo final - } \\
\text { Masculino }\end{array}$} & \multicolumn{2}{|c|}{$\begin{array}{l}\text { Modelo final - } \\
\text { Feminino }\end{array}$} \\
\hline & $\mathrm{RC}$ & IC 95\% & $\mathrm{RC}$ & IC 95\% \\
\hline Idade & $1,00^{\mathrm{NS}}$ & $(0,97 ; 1,03)$ & $1,01^{\mathrm{NS}}$ & $(0,99 ; 1,04)$ \\
\hline \multicolumn{5}{|l|}{ Raça/cor (ref. Brancos) } \\
\hline Negros & $0,37^{\star \star \star}$ & $(0,23 ; 0,60)$ & $0,64^{\star}$ & $(0,45 ; 0,92)$ \\
\hline Amarelos/Indígenas & $5,16^{\star}$ & $(1,19 ; 22,37)$ & $0,33^{\mathrm{NS}}$ & $(0,10 ; 1,03)$ \\
\hline \multicolumn{5}{|l|}{ Região de moradia (ref. Sudeste) } \\
\hline Norte & $3,63^{\star \star}$ & $(0,17 ; 0,76)$ & $0,40^{\star \star \star}$ & $(0,22 ; 0,70)$ \\
\hline Nordeste & $3,91^{\star \star \star}$ & $(0,24 ; 6,43)$ & $0,36^{\star \star \star}$ & $(0,23 ; 0,54)$ \\
\hline Sul & $0,60^{\mathrm{NS}}$ & $(0,32 ; 1,14)$ & $0,64^{\mathrm{NS}}$ & $(0,38 ; 1,07)$ \\
\hline Centro-Oeste & $0,63^{\mathrm{NS}}$ & $(0,34 ; 1,18)$ & $0,62^{\star}$ & $(0,39 ; 0,98)$ \\
\hline \multicolumn{5}{|l|}{$\begin{array}{l}\text { Escolaridade (ref. Até Ensino Fund. } \\
\text { Incompleto) }\end{array}$} \\
\hline Fund. Completo e Med. Incompleto & $2,15^{\mathrm{NS}}$ & $(0,97 ; 4,76)$ & $2,59^{\star \star \star}$ & $(1,59 ; 4,20)$ \\
\hline Med. Completo e Sup. Incompleto & $3,30^{\star \star \star}$ & $(1,69 ; 6,42)$ & $3,181^{\star \star}$ & $(1,50 ; 6,72)$ \\
\hline Superior completo & $28,15^{\star \star \star}$ & $(9,81 ; 80,80)$ & $6,06^{\star \star \star}$ & $(3,01 ; 12,20)$ \\
\hline \multicolumn{5}{|l|}{$\begin{array}{l}\text { Consumo de bebidas alcóolicas (ref. } \\
\text { Não consome) }\end{array}$} \\
\hline Menos de 1 vez por mês & $1,35^{\mathrm{NS}}$ & $(0,75 ; 2,42)$ & $3,14^{\star \star \star}$ & $(1,68 ; 5,87)$ \\
\hline Uma ou mais vezes por mês & $3,35^{\star \star \star}$ & $(2,04 ; 5,50)$ & $1,87^{\mathrm{NS}}$ & $(0,87 ; 4,04)$ \\
\hline \multicolumn{5}{|l|}{ Tabagismo (Nunca fumou) } \\
\hline Fumante & $0,48^{*}$ & $(0,27 ; 0,86)$ & - & - \\
\hline Ex-fumante & $0,85^{\mathrm{NS}}$ & $(0,50 ; 1,45)$ & - & - \\
\hline \multicolumn{5}{|l|}{$\begin{array}{l}\text { Atividades religiosas (ref. } \\
\text { Frequentemente) }\end{array}$} \\
\hline Não frequenta & $0,52^{*}$ & $(0,32 ; 0,85)$ & $0,40^{\star \star \star}$ & $(0,27 ; 0,58)$ \\
\hline Raramente & $0,73^{\text {NS }}$ & $(0,40 ; 1,33)$ & $0,570^{\star \star}$ & $(0,38 ; 0,86)$ \\
\hline \multicolumn{5}{|l|}{$\begin{array}{l}\text { Doenças crônicas não transmissiveis } \\
\text { (ref. Nenhuma doença) }\end{array}$} \\
\hline Uma doença & $0,23^{\star \star \star}$ & $(0,13 ; 0,40)$ & $0,32^{\star \star \star}$ & $(0,19 ; 0,52)$ \\
\hline Duas a três doenças & $0,11^{\star \star \star}$ & $(0,06 ; 0,20)$ & $0,08^{\star \star \star}$ & $(0,54 ; 0,13)$ \\
\hline Quatro a mais doenças & $0,02^{\star \star \star}$ & $(0,01 ; 0,05)$ & $0,02^{\star \star \star}$ & $(0,10 ; 0,35)$ \\
\hline \multicolumn{5}{|l|}{$\begin{array}{l}\text { Capacidade funcional (ref. } \\
\text { Independente) }\end{array}$} \\
\hline Dependente AVD e AIVD & $0,07^{\star \star \star}$ & $(0,02 ; 0,19)$ & $0,37^{\star}$ & $(0,18 ; 0,74)$ \\
\hline Dependente AIVD & $0,09^{\text {NS }}$ & $(0,01 ; 1,14)$ & $0,10^{\star \star \star}$ & $(0,03 ; 0,36)$ \\
\hline
\end{tabular}

Nota: Significância estatística: * valor de $p<0,05 ;{ }^{* \star}$ valor de $p<0,01$; *** valor de $p<0,001$; NS: não significativo. 
e Nordeste, as chances foram maiores de autopercepção da saúde positiva, quando comparados com aqueles que residem na Regiáo Sudeste $(\mathrm{RC}=3,63 \mathrm{e}$ 3,91 , respectivamente). Quanto às mulheres, os dados mostraram que as idosas residentes nas regióes Norte e Nordeste apresentaram menos chances de perceberem a saúde de forma positiva, sendo $60 \%$ e $64 \%$, respectivamente, a menos de chances do que para aquelas que residiam no Sudeste. Com relação à escolaridade, observa-se tanto para idosos do sexo masculino quanto do que sexo feminino que, quanto maior o nível educacional, menor a chance de percepção negativa. Idosos com ensino médio completo e superior incompleto obtiveram três vezes maiores chances de percepção a saúde positivamente quando comparados com os idosos com escolaridade inferior ao nível fundamental completo (Tabela 4). Quanto ao estilo de vida (Tabela 4), nunca ter fumado e ter participado de atividades de interação social religiosa com maior frequência (categorias de referência no modelo) apresentaram chances maiores de avaliação positiva da saúde para homens e mulheres ( $\mathrm{RC}$ menores que zero quando comparadas às categorias de referência). Por outro lado, o uso de alguma quantidade de bebida alcoólica, sendo em maior quantidade para os homens $\left(\mathrm{RC}_{\text {uma ou mais vezes no mês }}=3,35\right)$ do que para as mulheres $\left(\mathrm{RC}_{\text {menos de uma vez ao mês }}=3,14\right)$, contribuem para uma chance maior de declarar a saúde como muito boa/ boa. Conforme esperado, em ambos os sexos, não apresentar incapacidades ou DCNT são fatores que contribuem para maiores chances de autoavaliação positiva da saúde.

\section{Discussão}

Os resultados evidenciam os pressupostos teóricos adotados pelo estudo, baseados nos determinantes sociais de saúde que incluíram as divisões de estratificação social e as hierarquias de poder baseadas nas desigualdades de gênero. Ser homem, da cor autodeclarada branca, possuir maior escolaridade, apresentar menor numero de DCNT e capacidade funcional preservada que permita a independência nas AVD e AIVD foram variáveis associadas a uma melhor percepção da saúde. Perceber a saúde positivamente parece refletir uma posição social e os mecanismos estruturais que organizam os contextos socioeconômicos ao longo do curso da vida.

Neste estudo, a percepção positiva da saúde com número relativo de homens superior ao de mulheres para avaliaçóes muito boas e boas da saúde corrobora com pesquisas relacionadas as questôes de gênero e o uso desigual do tempo laboral entre homens e mulheres ao longo do curso de vida, ${ }^{(16)}$ pois a percepção está associada a fatores biológicos, sociais e comportamentais ancorados na perspectiva teórica dos determinantes sociais de saúde. ${ }^{(5,14)}$ No entanto, outros estudos não identificaram diferenças de percepção de saúde entre homens e mulheres. ${ }^{(17,18)}$

Um total de sete em cada dez pessoas idosas não tinha completado o ensino fundamental. Este dado é importante, pois o nível de escolarização também foi apontado como um determinante significativo na percepção de saúde: idosos com maior escolaridade têm menos chances de avaliaçóes negativas. ${ }^{(17)}$ A posição social contempla as condiçôes materiais da vida e a escolaridade irá impactar o capital social de cada idoso e seu estilo de vida, seu acesso aos serviços de saúde e a qualidade do tratamento das DCNT. A baixa escolaridade está relacionada a piores condiçôes de saúde estando também associada à autopercepção negativa da saúde. ${ }^{(19,20)}$ Ressalta-se que o percentual de idosos com percepção positiva diminuiu conforme a idade e o número de DCNT aumentava. ${ }^{(18)}$ Outro estudo apontou que idosos com nenhum ano de escolaridade apresentaram mais chances de avaliar negativamente sua saúde do que aqueles com mais de oito anos. ${ }^{(20)} \mathrm{O}$ mesmo estudo demonstrou que a depender do estilo de vida, as idosas que não tinham o hábito regular de práticas religiosas perceberam negativamente sua saúde, ao contrário daquelas que frequentavam regularmente. A prática de atividades religiosas foi significativa apenas para as mulheres. A religião e a cultura podem interferir nos comportamentos, relacionando-se com consumo de bebidas, tabagismo e outras questóes alimentares. É importante destacar que, antes de ser apenas uma prática religiosa, a presença frequente em grupos proporciona uma ampliaçáo da rede social da pessoa idosa e represen- 
ta uma atividade de interação social e possivelmente uma atividade de enfrentamento ao isolamento social relatado nessa etapa da vida. ${ }^{(21)}$

As variáveis de condição de saúde foram as que apresentaram maior prevalência na autopercepção de saúde. Para ambos os sexos, a presença de DCNT e a incapacidade funcional influenciaram na percepção negativa da saúde. Estas variáveis tiveram maior associação para as idosas. A presença de doenças permite uma associação com autoavaliação de saúde negativa, pois as polimorbidades podem trazer limitaçôes e dificuldades de autocuidado, o que pode proporcionar a autopercepção negativa de saúde do idoso. ${ }^{(18,22)}$

Idosos do sexo masculino residentes nas regióes Norte e Nordeste, que possuíam menor escolaridade, que tinham mais DCNT, eram dependentes nas AVD e nas AIVD ou apenas nas últimas, foram aqueles que mais perceberam negativamente sua saúde. Assim como, as mulheres com 60 anos ou mais, residentes nas regióes Norte e Nordeste, com menor escolaridade, que não frequentavam atividades religiosas, possuíam DCNT ou eram dependentes nas AVD e nas AIVD foram as que mais avaliaram sua saúde de forma negativa.

O perfil das pessoas idosas que se apresentou associado à autoavaliação positiva indica que a saúde da população idosa é definida por determinantes que se aproximam do conceito ampliado de saúde como resultado de todas políticas, além de reforçar a importância do direito a educação de pessoas idosas como componente da promoçáo da saúde dessa população e prevenção dos agravos relacionados às DCNT e incapacidades. Um estudo anterior já identificava a associação entre as atividades de vida diária, capacidade de auto referenciar doenças e a escolaridade. ${ }^{(23)}$ Uma base de dados que permitisse uma análise dos fatores relacionados à síndrome da fragilidade e seus diferenciais para mulheres e homens idosos poderia auxiliar em um detalhamento mais preciso acerca do impacto das DCNT na percepção de saúde. ${ }^{(24,25)}$ Entretanto é recomendável a padronização das perguntas e respostas de pesquisa sobre auto avaliação de saúde, pois essas informaçôes possibilitarão conhecer, acompanhar e compa- rar resultados para orientar a tomada de decisóes no tocante à formulação de políticas públicas de saúde.

Adicionalmente, outras limitaçóes merecem ser pontuadas. A base de dados utilizada não inclui indivíduos institucionalizados; ${ }^{(26)}$ está sujeita a efeitos de seleção, ou seja, as informaçóes coletadas pela pesquisa e analisadas neste estudo se referem apenas aos idosos sobreviventes; e, que as informações foram fornecidas por meio de auto relato (o próprio idoso foi o informante) e, portanto, as respostas podem ser influenciadas por fatores culturais, de memória, pela escolaridade ou por questôes psicológicas e emocionais. Esses fatores podem enfraquecer ou fortalecer os efeitos dos parâmetros estimados nos modelos logísticos.

Finalmente, espera-se que os resultados apresentados tragam um maior conhecimento dos fatores associados à auto avaliação da saúde como positiva pelas pessoas idosas, bem como permita identificar pontos que merecem atenção no planejamento e na implementação de políticas públicas e que podem contribuir para ganhos de vida com mais qualidade.

\section{Conclusão}

O estudo identificou os determinantes sociais de saúde dos idosos e a relação com uma percepçáo positiva da saúde. Identificar e analisar estas associaçôes são pontos importantes para a elaboração de políticas públicas específicas, visando a equidade e a promoção da saúde. Políticas públicas e planos de cuidado de profissionais da saúde voltados para decifrar a determinação social da saúde e planejar açóes integradas que promovam o envelhecimento ativo e saudável podem contribuir para uma autopercepçáo mais positiva da saúde por parte da população idosa. A autopercepção de saúde é uma ferramenta que permite identificar a percepção do idoso em relação ao significado da sua saúde, que envolve subjetividades, julgamentos, emoçóes, perspectivas e oportunidades, mas acima de tudo expressa o gradiente social e as desigualdades sociais e de gênero e tem se consolidado como um instrumento relevante para a análise de demanda por saúde. 


\section{Agradecimentos}

\author{
Agradecemos à Coordenação de Aperfeiçoamento \\ de Pessoal de Nível Superior pela bolsa PNPD para \\ RCSC.
}

\section{Colaborações}

Gomes MMF, Paixão LAR, Faustino AM, Cruz RCS e Moura LBA contribuíram com a concepção do projeto, análise e interpretação dos dados, redação do artigo, revisão crítica relevante do conteúdo intelectual e aprovação da versão final a ser publicada.

\section{Referências}

1. World Health Organization (WHO). World report on ageing and health. Geneva: WHO; 2015.

2. Geib LT. Determinantes sociais da saúde do idoso. Ciênc Saúde Coletiva. 2012;17(1):123-32.

3. Tkatch R, Musich S, MacLeod S, Alsgaard K, Hawkins K, Yeh CS. Population health management for older adults: review of interventions for promoting successful aging across the health continuum. Gerontol Geriatr Med. 2016;2(2):2333721416667877.

4. Lima-Costa MF, Firmo J0, Uchôa E. Differences in self-rated health among older adults according to socioeconomic circumstances: the Bambuí Health and Aging Study. Cad Saude Publica. 2005;21(3):8309.

5. Confortin SC, Giehl MW, Antes DL, Schneider IJ, d'Orsi E. Autopercepção positiva de saúde em idosos: estudo populacional no Sul do Brasil. Cad Saude Publica. 2015;31(5):1049-60.

6. Blazer DG. How do you feel about...? Health outcomes in late life and self-perceptions of health and well-being. Gerontologist. 2008;48(4):415-22.

7. Pérez-Zepeda MU, Belanger E, Zunzunegui MV, Phillips S, Ylli A, Guralnik $J$. Assessing the validity of self-rated health with the short physical performance battery: a cross-sectional analysis of the International Mobility in Aging Study. PLoS One. 2016;11(4):e0153855.

8. Babones SJ. The consistency of self-rated health in comparative perspective. Public Health. 2009;123(2):199-201.

9. Lima-Costa MF, Cesar CC, Chor D, Proietti FA. Self-rated health compared with objectively measured health status as a tool for mortality risk screening in older adults: 10-year follow-up of the Bambuí cohort study of aging. Am J Epidemiol. 2012;175(3):228-35.

10. Bendayan R, Piccinin AM, Hofer SM, Muniz G. Are changes in self-rated health associated with memory decline in older adults? J Aging Health. 2017;29(8):1410-23.
11. Instituto Brasileiro de Geografia e Estatística (IBGE). Pesquisa Nacional de Saúde 2013: percepção do estado de saúde, estilos de vida e doenças crônicas [Internet]. Rio de Janeiro: IBGE; 2014. [citado 20220 Mai 31]. Disponível em: https://saudeamanha.fiocruz.br/wp-content/ uploads/2017/03/Pesquisa-Nacional-de-Sau\%CC\%81de-2013percepc\%CC\%A7a\%CC\%830-do-estado-de-sau\%CC\%81de-estilosde-vida-e-doenc\%CC\%A7as-cro\%CC\%82nicas.pdf

12. Zanesco C, Bordin D, Santos CB, Müller EV, Fadel CB. Factors determining the negative perception of the health of Brazilian elderly people. Rev Bras Geriatr Gerontol. 2018;21(3):283-92.

13. Carneiro JA, Gomes CA, Durães W, Jesus DR, Chaves KL, Lima CA, et al. Autopercepção negativa da saúde: prevalência e fatores associados entre idosos assistidos em centro de referência. Cien Saude Colet. 2018:909-18.

14. Marmot M, Bell R. Social determinants and non-communicable diseases: time for integrated action. BMJ. 364:1251.

15. Ricardo RC, Fermino RC, Hallal PC, Reis RS. Validade e fidedignidade da escala de satisfação com a prática de atividade física em adultos. Rev Saude Publica. 2011;45(2):286-93.

16. Bandeira LM, Preturlan RB. As pesquisas sobre uso do tempo e a promoção da igualdade de gênero no brasil. In: Fontoura N, editor. Araújo C, organizadores. Uso do tempo e gênero. Rio de Janeiro: $A B E$ Graph Gráfica e Editora Ltda; 2016.

17. Robert SA, Cherepanov D, Palta M, Dunham NC, Feeny D, Fryback DG. Socioeconomic status and age variations in health-related quality of life: results from the national health measurement study. J Gerontol B Psychol Sci Soc Sci. 2009;64(3):378-89.

18. Souza MS, Coqueiro RS, Fernandes MH. Estudo populacional sobre os determinantes da autopercepção de saúde de idosos residentes em comunidade. Cienc Enferm. 2016;22(2):13-26.

19. Nunes AP, Barreto SM, Gonçalves LG. Relações sociais e autopercepção da saúde: projeto envelhecimento e saúde. Rev Bras Epidemiol. 2012;15(2):415-28.

20. Pagotto V, Bachion MM, Silveira EA. Autoavaliação da saúde por idosos brasileiros: revisão sistemática da literatura. Rev Panam Salud Publica. 2013;33(4):302-10.

21. Domènech-Abella J, Mundó J, Haro JM, Rubio-Valera M. Anxiety, depression, loneliness and social network in the elderly: Longitudinal associations from The Irish Longitudinal Study on Ageing (TILDA). J Affect Disord. 2019;246:82-8.

22. Borim FS, Francisco PM, Neri AL, Barros MB. Dimensões da autoavaliação de saúde em idosos, São Paulo, Brasil. Cad Saude Publica. 2014;48(5):714-22.

23. Araújo GK, Souto RQ, Alves FA, Sousa RC, Ceballos AG, Santos RC, et al. Capacidade funcional e fatores associados em idosos residentes em comunidade. Acta Paul Enferm. 2019;32(3):312-8.

24. Borim FS, Francisco PM, Neri AL. Fatores sociodemográficos e de saúde associados à mortalidade em idosos residentes na comunidade. Rev Saude Publica. 2017;51:42.

25. Buckinx F, Rolland Y, Reginster JY, Ricour C, Petermans J, Bruyère 0. Burden of frailty in the elderly population: perspectives for a public health challenge. Arch Public Health. 2015;73(1):19-26.

26. Szwarcwald CL, Malta DC, Pereira CA, Vieira ML, Conde WL, Souza Júnior PR, et al. Pesquisa Nacional de Saúde no Brasil: concepção e metodologia de aplicação. Cien Saude Colet. 2014;19(2):333-42. 